\title{
HEAT TREATMENT OF CdSe THIN FILMS AND APPLICATION TO PHOTOPOTENTIOMETERS
}

\author{
MASUJI SATO, SATORU KAWAI $\dagger$ and FUMIAKI YAMADA
}

\author{
Fujitsu Limited, †Fujitsu Laboratories Ltd., 1015, Kamikodanaka, Nakaharaku, Kawasaki, 211, Japan
}

\begin{abstract}
Effects of heat treatment have been studied in a nitrogen atmosphere for improving the characteristics of poor photoconductive CdSe evaporated films. The following results were obtained. At first light resistance and dark resistance greatly decrease after the heat treatment of evaporated films in a pure nitrogen atmosphere for an hour. The ratio of dark resistance to light resistance under irradiation of 1,000/ux remained as low as an order of magnitude of 10 . Secondary increase of an order of magnitude of $10^{6}$ or $10^{7}$ in dark resistance is obtained, adding to the post treatment in $\mathrm{N}_{2}+\mathrm{O}_{2}$ atmosphere for thirty seconds after the main heat treatment in $\mathrm{N}_{2}$ for an hour. The ratio of dark to light resistance becomes an order of $10^{8}$ and as for photoresponse, both rise and decay time of photocurrent become $0.5 \mathrm{~ms}$. The results indicates that heat treated films have characteristics of good photosensitivity and quick response in photocurrent.

The photopotentiometer has been analysed by using the transmission line theory and device input-output characteristics have been related to the properties of the resistive film and photoconductive film of the device.

The photopotentiometer using the improved photoconductive CdSe thin film has been fabricated and examined. A good independent linearity of less than $2 \%$ at a speed of $2000 \mathrm{rpm}$ is obtained.
\end{abstract}

\section{INTRODUCTION}

High speed input-output devices are required to speed up information processing machines. The potentiometer which is a mechanical-electrical conversion device is indispensable as a servoelement in servomotors. However, contact type potentiometers may cause inferior contact as chattering, burning and speeding-up will lower reliability. Non-contact type potentiometers will resolve these problems.

The authors have made a non-contact type potentiometer using photoconductive thin film and part has been reported. ${ }^{1}$ It is necessary to fabricate good photoconductive and quick photoresponsive film to realize a photopotentiometer for use as a servoelement, Thus effects of heat treatment have been studied for improving the characteristics of poor photoconductive CdSe evaporated films and a new method of heat treatment has been found.

Device analysis using the transmission line theory has been done to study the electronic mechanism of photopotentiometers. Device input-output characteristics have been related to the properties of the resistive film and photoconductive film of the device.

The photopotentiometer for sensing vibration specially was made on an experimental basis by combining this photoconductive film and a $\mathrm{NiCr}$ resistive film. It was confirmed that the potentiometer responded to $2,000 \mathrm{rpm}$ which conventional contact type potentiometers could not easily follow.

\section{DESIGN OF PHOTOPOTENTIOMETER}

\subsection{Analysis}

The photopotentiometer has the structure shown in Figure 1. The linear field is applied in the resistive film by input current $I_{0}$ and light spot (width $\Delta S$ ) is irradiated on position $S$ of the photoconductive film in this element. The resistance of the portion irradiated by the light spot is converted to low resistive value $R p / \Delta S$ from semi-insulating value $R d / \Delta S$ and the output voltage $S V_{0}$ is produced at the output electrode. The position $S$ is changed from 0 (ground) to 1 (input electrode) as a normalized value, so the output voltage changes linearly according to light spot position $S$.

The equivalent circuit for device analysis is shown in Figure 2. The circuit is the distributed constant line. According to the circuit, the equations giving input resistance $R i(S)$ and output voltage $V e(S)$ are induced 


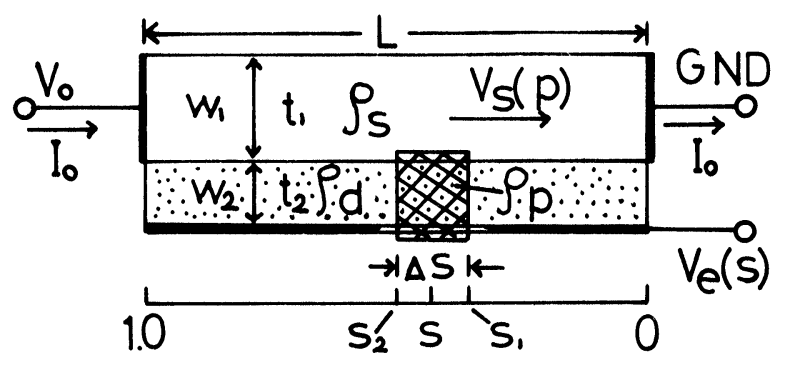

Normalized length $P$

Resistive film

$R s=\rho_{s} L / t_{1} w_{1}=r_{s} L / w_{1}$

Photoconductive film

Dark resistance

$R_{d}=\rho_{\mathrm{aW}} \mathrm{w}_{2} / \mathrm{t}_{2} \mathrm{~L}=\mathrm{r}_{\mathrm{d}} / \mathrm{t}_{2} \mathrm{~L}$

Light resistance

$R_{p}=r_{p} / t_{2} L=R_{d} / k^{2}$

\section{Electrode film}

Portion irradiated bylightspot

$$
S=S_{1}+\Delta S / 2=S_{2}-\Delta S / 2
$$

\section{$V_{S}(p):$ Potential distribution in resistive film}

FIGURE 1 Construction of a photopotentiometer showing the various regions.

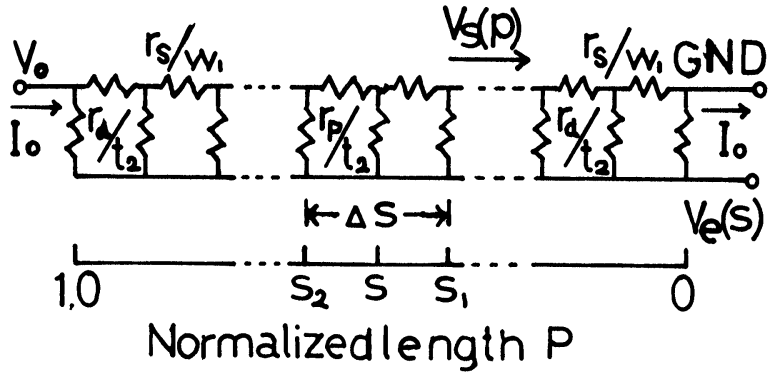

FIGURE 2 Equivalent circuit of a photopotentiometer. as shown in Eqs. (1)-(4). Eqs. (2) and (4) are approximate equations so far as $\alpha \ll 1$ and $\kappa^{2} \gg 1$.

$$
\begin{aligned}
& \frac{R i(S)}{R S}=\frac{2}{\alpha} \frac{[X]}{[Y]} \\
& \simeq 1-\Delta S+\frac{\gamma s p}{R s} \frac{\sinh \kappa \alpha}{\sinh \kappa \alpha \Delta S} \frac{\cosh \kappa \alpha \Delta S-1}{\cosh \kappa \alpha-1} \\
& {[X]=\cosh \alpha(1-\Delta S) \cosh \kappa \alpha \Delta S} \\
& +\frac{1}{2}\left(\kappa+\frac{1}{\kappa}\right) \sinh \alpha(1-\Delta S) \times \sinh \kappa \alpha \Delta S-1 \\
& {[Y]=\sinh \alpha(1-\Delta S) \cosh \kappa \alpha \Delta S+} \\
& \left\{\begin{array}{l}
\left\{\cosh \alpha S_{1} \cosh \alpha\left(1-S_{2}\right)+\frac{1}{\kappa} \cosh \alpha S_{1} \sinh \alpha\left(1-S_{2}\right)\right\} \\
\sinh \kappa \alpha \Delta S
\end{array}\right. \\
& \frac{V e(S)}{V_{0}}=\frac{[U]}{[V]} \\
& \simeq S \\
& {[U]=\cosh \alpha\left(1-S_{2}\right)-\frac{R s}{R i(S) \alpha}\left\{\sinh \alpha\left(1-S_{2}\right)\right.} \\
& \left.+\sinh \alpha S_{1} \cosh \kappa \alpha \Delta S\right\} \\
& {[V]=\cosh \alpha\left(1-S_{2}\right)-\left\{\cosh \kappa \alpha \Delta S-\frac{1}{\kappa} \sinh \alpha S_{1}\right.} \\
& \sinh \kappa \alpha \Delta S\}
\end{aligned}
$$

where $\gamma_{s p}$ is the input resistance of a photo-

potentiometer element, the whole surface of which is irradiated by the light and is given by Eq. (5). $\alpha$ and $\kappa \alpha$ are the propagation constants of the transmission lines shown in Figure 2 and are given in $\sqrt{ } R s / R d$ and $\sqrt{ } R s / R p .^{2}$

$$
\frac{\gamma s p}{R s}=\frac{2}{\kappa \alpha} \frac{\cosh \kappa \alpha-1}{\sinh \kappa \alpha}
$$

The dependence of value $\alpha$ on the voltage distribution $V s(P)$ in the resistive film is shown in Figure 3. $V s(P)$ is not a straight line because of degradation by dark resistance $R d . R d / R s \leqq 400$ is necessary to obtain a linearity of less than $0.1 \%$ on voltage distribution $V s(P)$.

The output voltage change is shown in Figure 4 when $\Delta S$ is fixed and $R d / R p\left(\kappa^{2}\right)$ is changed. One feature is that output voltage varies according to the value of $R d / R p . R d / R p \geqq 5000$ is necessary to obtain an output voltage of more than $95 \%$. 


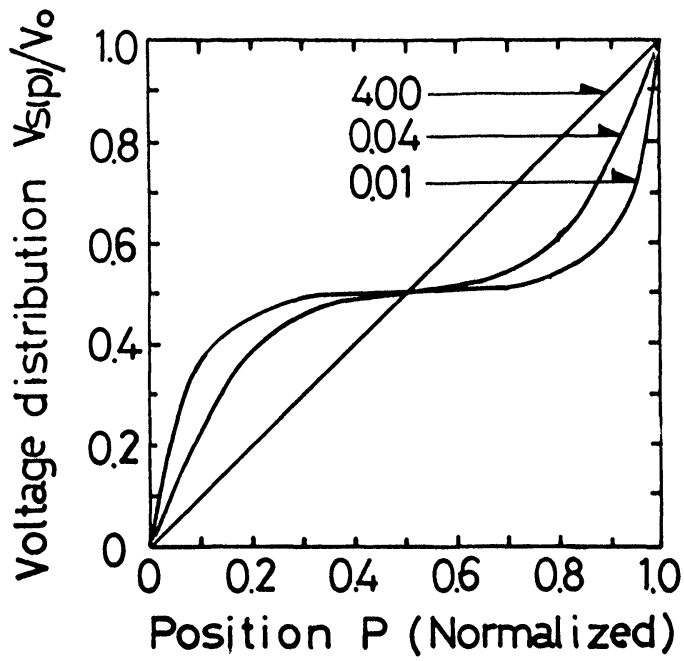

FIGURE 3 Variation of voltage distribution $V s(P)$ in value $R d / R s\left(\alpha^{-2}\right)$ without light spot.

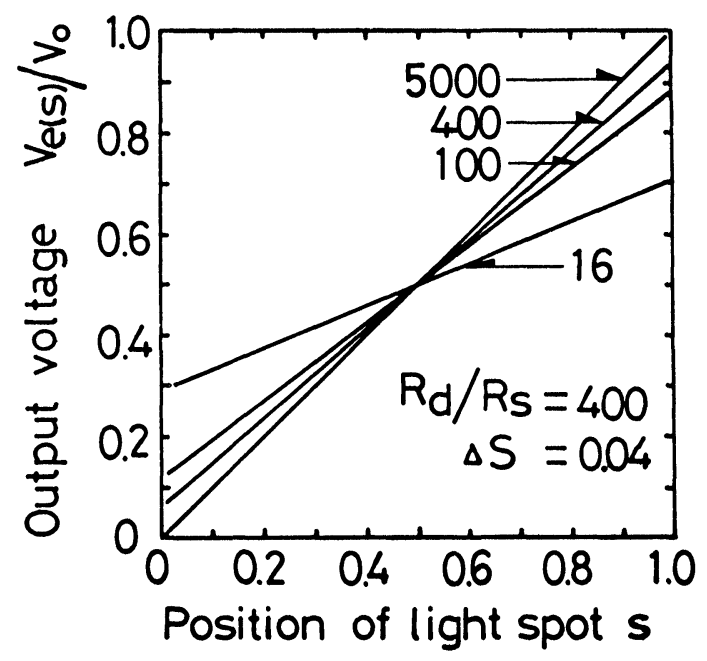

FIGURE 4 Variation of output voltage $V e(S)$ in value $R d / R p\left(\kappa^{2}\right)$.

\subsection{Electronic Mechanism}

The change of the current flowing in the resistive film when the irradiation of the light spot was or was not present has been considered. The effect is shown in Figure 5. Without light irradiation, the dark resistance, $R d$, of the photoconductive film is many orders greater than the resistance of $R s$ of the resistive film, so that the current flows in the resistive film only and the output voltage is not observed at the ouput electrode.

With the irradiation of the light spot on the position $S$
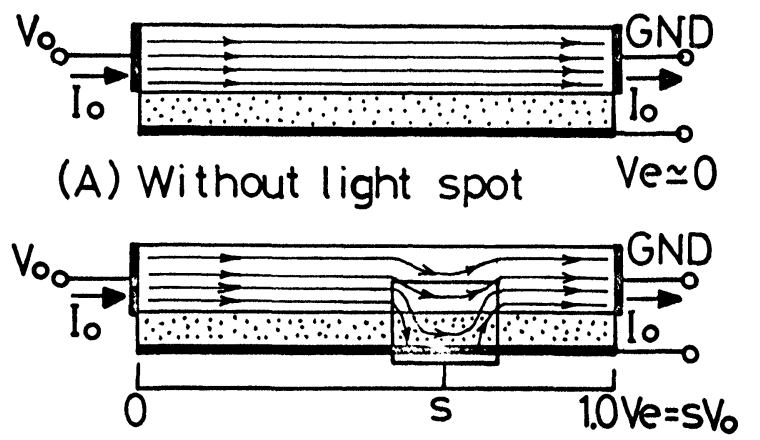

(B) With light spot

FIGURE 5 Electronic mechanism of a photopotentiometer.

of the photoconductive film, the resistance of the portion irradiated by the light spot decreases by some order, so that part of the current flows to the output electrode as shown in Figure 5 (B) and the output voltage $S V_{0}$ is produced at the output electrode. This is the electronic mechanism of the non-contact type potentiometer using the photoconductive effect.

The characteristics required of resistive film and photoconductive film are given in Eqs. (6), (7), (8) and (9).

$$
\begin{aligned}
& R d / R s \geqq 400 \\
& R d / R p \geqq 5000 \\
& R d \geqq 100(\mathrm{M} \Omega) \\
& R s, R p / \Delta S \leqq 10(\mathrm{k} \Omega)
\end{aligned}
$$

In these conditions, load resistance is defined as $10 \mathrm{M} \Omega$. Eqs. (8) and (9) are necessary so that output voltage is not observed without light irradiation and a loading error of less than 0.1 percent may be obtained.

\section{HEAT TREATMENT OF CdSe THIN FILMS}

Thin CdSe photoconductive film was selected for sensing red light and for quick response compared with CdS photoconductive film for the photopotentiometer. Thin CdSe films previously has poor photoconductivity when being formed by evaporation or sputtering. It was known already that heat treatment in an inert gas was necessary for improving photoconductivity. ${ }^{4} \mathrm{On}$ the basis of this knowledge, heat treatment of CdSe evaporated films was done in a nitrogen atmosphere. The film thickness was fixed at $7000 \pm 3000 \AA$.

The following results were obtained. The dark resistance of poor photoconductive and semi-insulating CdSe thin films greatly decrease for one hour. The ratio 

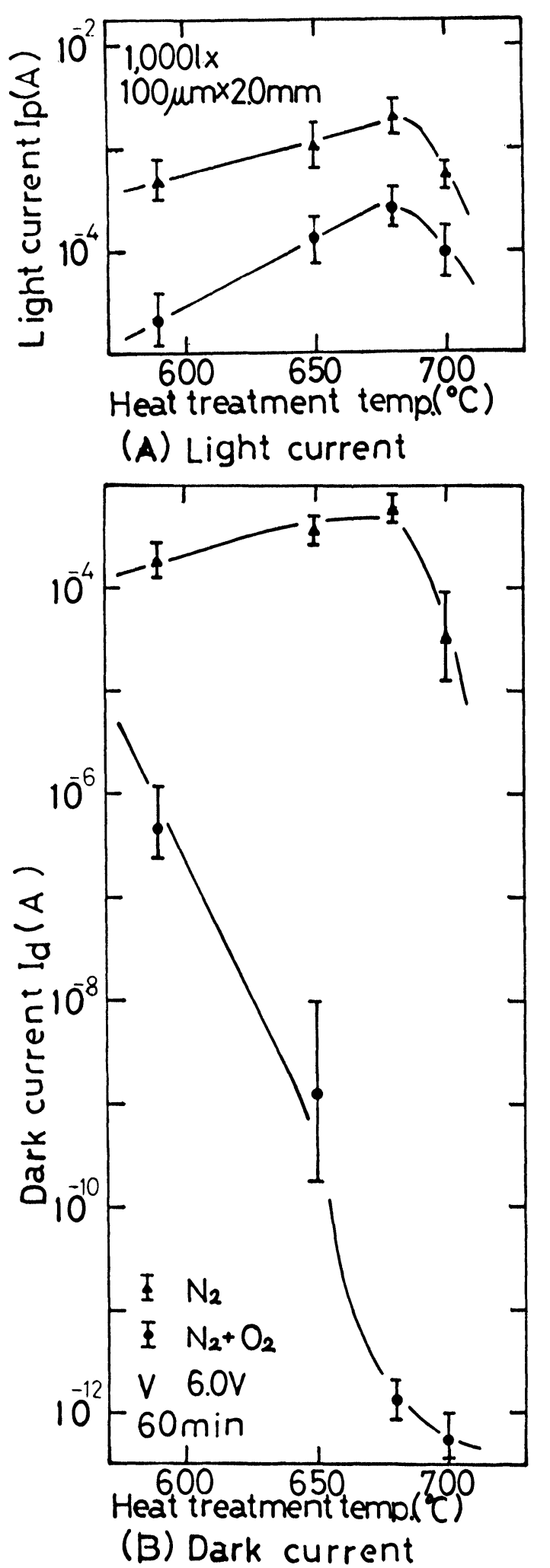

FIGURE 6 Variation of light current $I p$ and dark current $I d$ with heat treatment temperature.
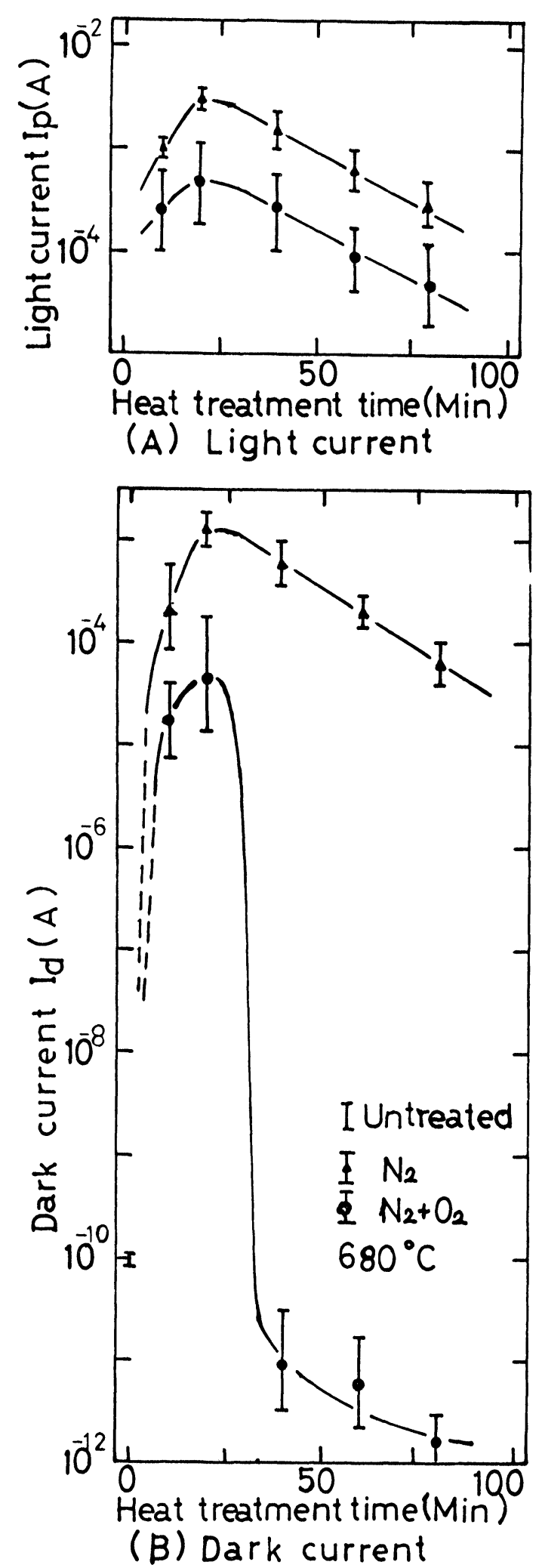

FIGURE 7 Variation of light current $I p$ and dark current Id with heat treatment time. 
of dark resistance to light resistance under irradiation of 1,000 lux remained as low as an order of magnitude of 10. Light resistance is the lowest and is suitable, but for low dark resistance is not suitable for use in a photopotentiometer.

Some methods were tried to improve low dark resistance and found that heat treatment in an oxygen atmosphere affected dark resistance. Thus adding post-treatment in an $\mathrm{O}_{2}(37 \%)+\mathrm{N}_{2}(63 \%)$ atmosphere for thirty seconds after the main heat treatment in $\mathrm{N}_{2}$ for one hour, an increase of an order of magnitude of $10^{6}$ or $10^{7}$ in dark resistance was obtained. The ratio of dark to light resistance become of an order of magnitude of $10^{8}$. This high resistive and good photoconductive film is suitable for use in a photopotentiometer.

The effects of the heat treatment are shown in Figure 6 and Figure 7. Figure 6 shows the dependence of heat treatment temperature and Figure 7 the dependence of heat treatment time. The flow rate of introduced oxygen was defined at $1.5 \mathrm{l} / \mathrm{min}$. for 30 seconds.

Adding oxygen treatment, both photoresponse rise and decay times were greatly decreased. Quick photoresponse was achieved specially in heat treatment beyond $680^{\circ} \mathrm{C}$. The aspect is shown in Figure 8 . The estimation of photoresponse was done at $90 \%$ value of the rise time and $10 \%$ value of the decay time of the photocurrent pulse. Conversion to $63 \%$ and $37 \%$ values is obtained by multiplying by 0.4 . The value of $\tau \gamma_{90}$, $\tau d_{10} \leqq 2.0$ msec exhibits a photoresponse three times as quick as already resported values.

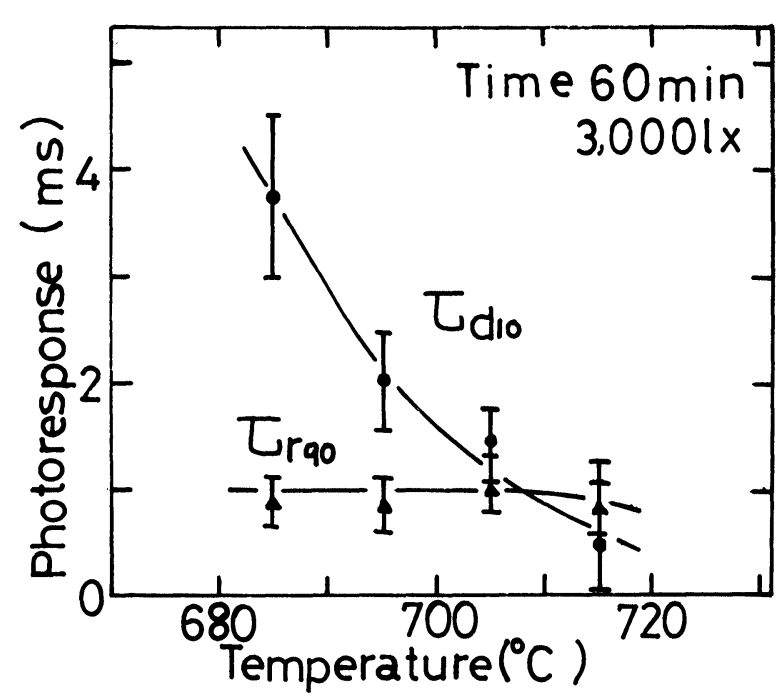

FIGURE 8 Variation of photoresponse $\tau_{\gamma 90}$ and $\tau_{d 10}$ with heat treatment temperature in CdSe films.

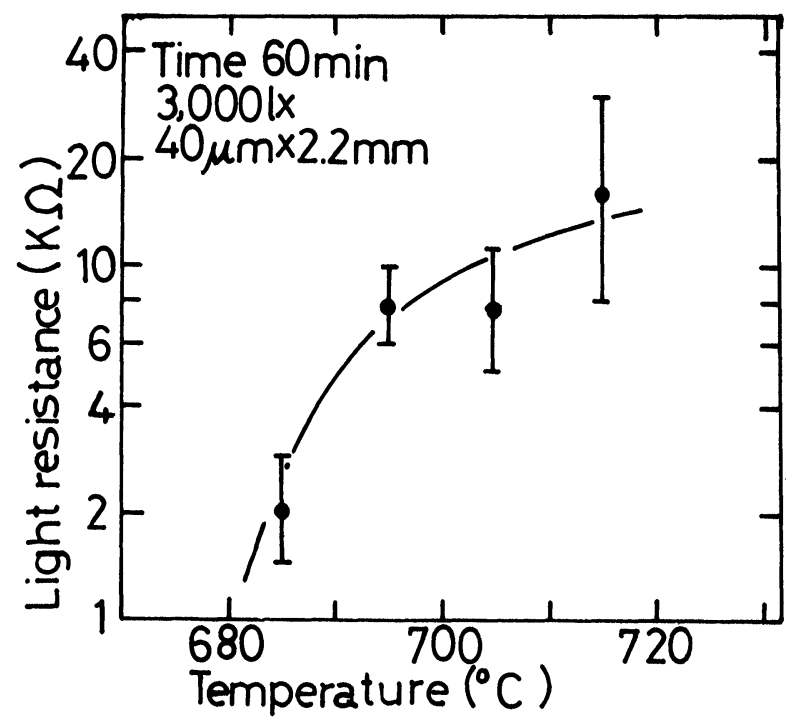

FIGURE 9 Variation of light resistance with heat treatment temperature in CdSe films.

Near these temperatures the increase of light resistance was observed as shown in Figure 9. Quick photoresponse film have a trend of large light resistance and dark resistance. The film to be employed in a photopotentiometer must have a light resistance of less than $10 \mathrm{k} \Omega$.

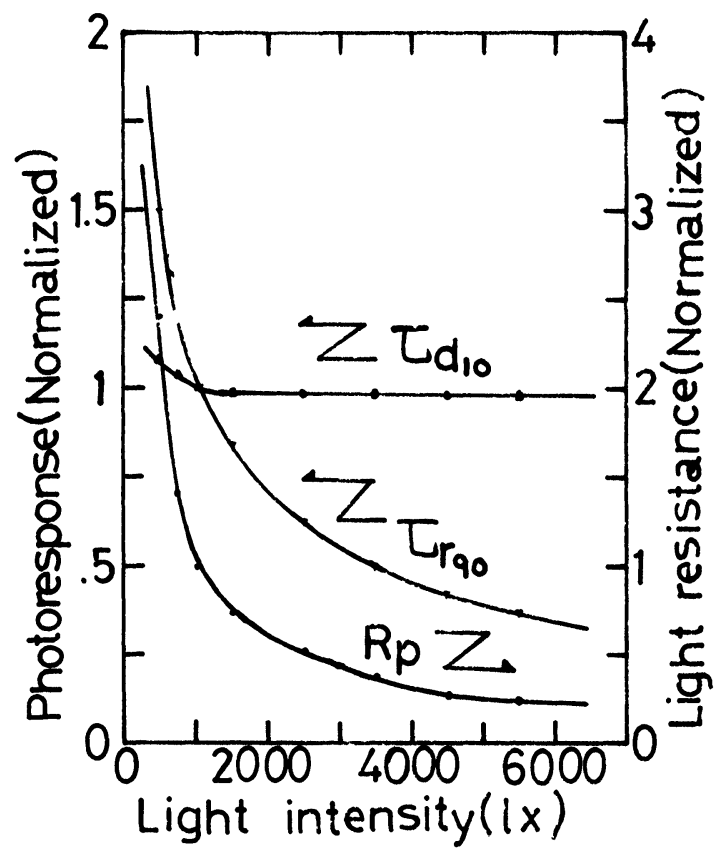

FIGURE 10 Variation of photoresponse and light resistance with light intensity. 
The dependence on light intensity of light resistance and photoresponse is shown in Figure 10. Increasing light intensity, light resistance decreases and at the same time, rise time decreases but decay time hardly changes. To decrease rise time by increasing light intensity contributes to speeding up of the photopotentiometer.

\section{APPLICATION OF PHOTOCONDUCTOR TO A POTENTIOMETER}

\subsection{Manufacturing}

Our product is a 90 degree type photopotentiometer which was developed for sensing vibrations mainly. The construction of the element is shown in Figure 11. The resistive was a $\mathrm{Ni}-\mathrm{Cr}$ film about $800 \AA$ thick and filmwidth was $200 \mu \mathrm{m}$. The film was made by vacuum evaporation. The electrode film was a $\mathrm{NiCr}-\mathrm{Au}$ film made by the vacuum evaporation method. The CdSe photoconductive film was about $0.7 \mu \mathrm{m}$ thick and the film width $\mathrm{W}_{2}$ was determined to be $40 \mu \mathrm{m}$ so that its equivalent brush resistance was less than $10 \mathrm{k} \Omega$. The element length was $11 \mathrm{~mm}$ and radius was $7.5 \mathrm{~mm}$.

The parts are shown in Figure 12. A super miniaturized lamp was used to provide a powerful light source. The lamp was operated at $80 \%(4.0 \mathrm{~V})$ of the fixed voltage. The average light intensity was $3,0001 \times$. These values satisfy Eqs. (6)-(9).

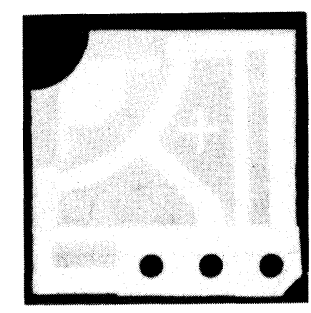

\section{$\square \mathrm{NiCr}$ resistive film \\ CaSe photoconductive film - NiCr-Au electrode film}

FIGURE 11 Construction of the element of a 90 degree type photopotentiometer.

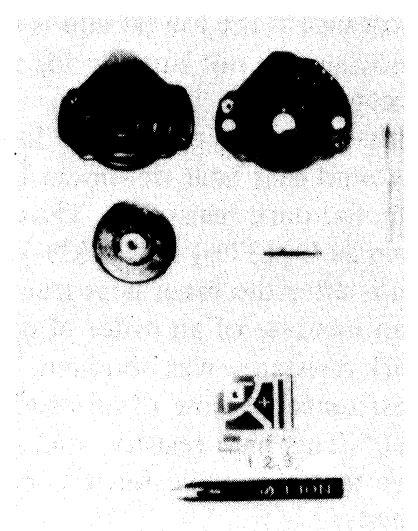

FIGURE 12 Parts of the photopotentiometer.

\subsection{Electrical Characteristics}

The angle-output voltage characteristic at static state is shown in Figure 13. Independent linearity was $\pm 1 \%$ for electric angles up to 50 degrees. The lowering in linearity near the input and ground electrodes is affected by running off the element's light spot. The

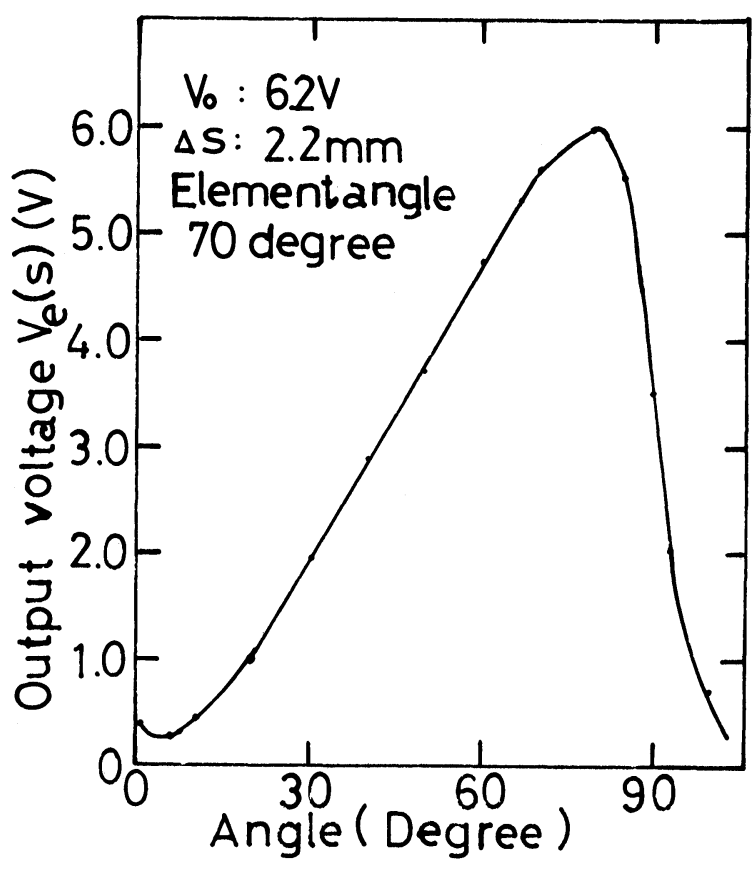

FIGURE 13 Angle-output voltage characteristic at static state. 
voltage output angle was about 100 degrees though the angle of the element was 70 degrees. This is affected by superimposing the ordinary output voltage upon the voltage of 14 degrees of light spot and the voltage of leakage light.

The characteristics of angle-input resistance and equivalent brush resistance are shown in Figure 14. Angular dependence on these values is affected by the angular distribution of light intensity. The large increase of equivalent brush resistance near the edges is affected by running off the element's light spot. The angular dependence of input resistance can be explained by (2).

The angle-output voltage characteristics at 200 and $2,000 \mathrm{rpm}$ are shown in Figure 15. The output voltage characteristic was straight for the angles without running off the element's light spot and output characteristic difference was small. Such a high speed response was not achieved easily in contact type potentiometers.

At high speeds it tends that the voltage production time becomes longer than the time which is defined by the revolution speed. This is affected by the photoresponse in the photoconductor. Running off the element's light spot creates an output voltage that is caused by residual light carrier. Especially, the decay time is longer than the rise time and the influence of

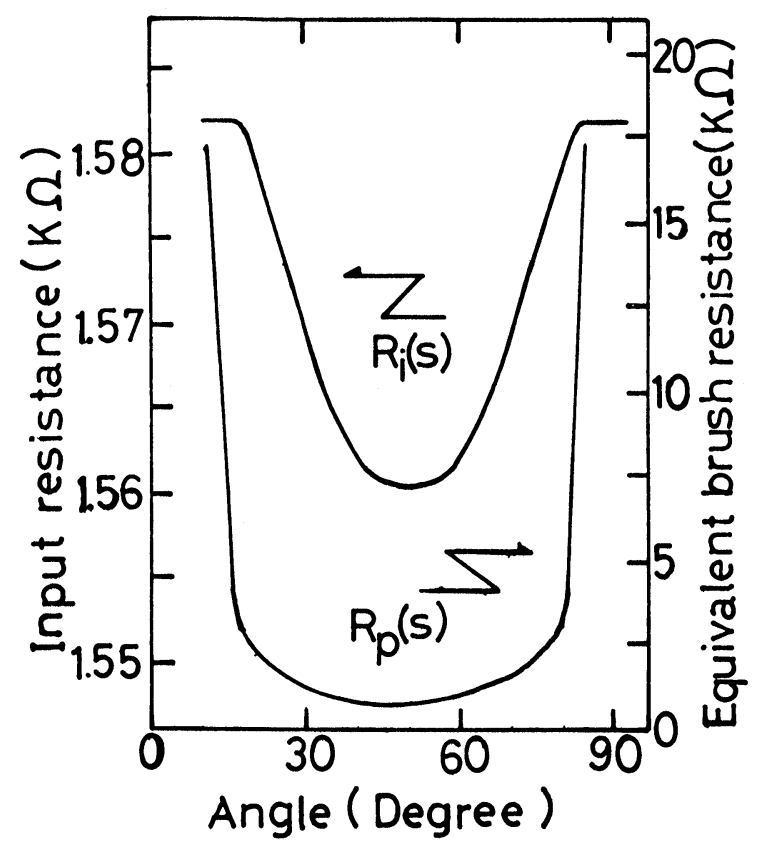

FIGURE 14 Angle-input resistance $\operatorname{Ri}(S)$ and equivalent brush resistance $R p(S)$ characteristics at static state.

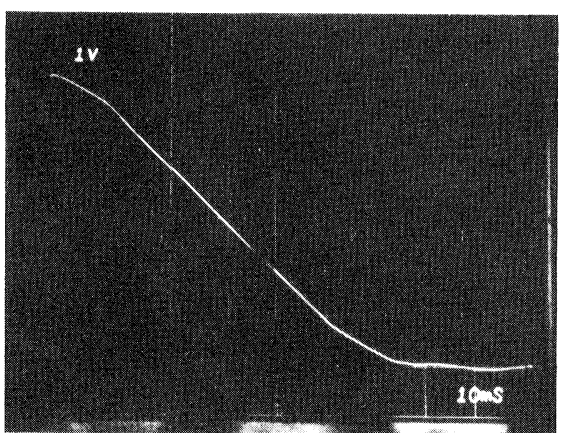

\section{(A) $200 \mathrm{rpm}$ (300ms/revolution)}

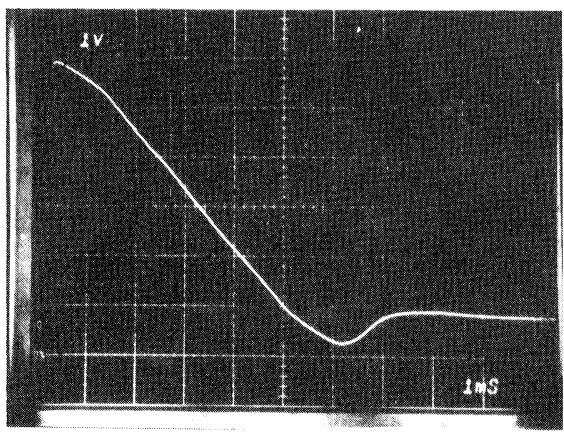

\section{(B) $2,000 \mathrm{rpm}(30 \mathrm{~ms} /$ revolution )}

FIGURE 15 Angle-output voltage characteristics at dynamic state (200 rpm and $2000 \mathrm{rpm})$.

decay time is remarkable. Therefore there is a small difference in the direction of revolution in the output voltage characteristic. To improve this weak point we will make efforts for a more quick photoresponse in photoconductors.

\section{CONCLUSION}

A non-contact type potentiometer employing the photoconductive effect was produced. This photopotentiometer responded to $2,000 \mathrm{rpm}$ and has been used as a servocontrol element.

Photopotentiometers, were studied and tried with the device analysis using the transmission line theory, and the electronic mechanism was cleared for the first time.

Further, a method of heat treatment was found which improved the poor photoconductive thin CdSe film so that it became useful as a photoconductive film. The improved film is indispensable for the fabrication of the quick response photopotentiometer. 


\section{ACKNOWLEDGEMENTS}

The authors wish to thank the staff of the Components Development Division of Fujitsu Limited for their support and Mr. N. Takagi for his good advice.

\section{REFERENCES}

1. N. Takagi, H. Yamada, M. Sato and K. Tasai, "A Fast
Response Non-contact Type Potentiometer Using An Improved CdSe Film", Proceedings of the 28th EEC, pp. 223-28 (1978).

2. H. Murrmann and D. Widmann "Current Crowding on Metal Contacts to Planar Devices", IEEE Trans. Electron Devices, pp. 1022-24 (1969).

3. M. Honda, M. Fuijmori and Y. Nishimura, "CdS Films by RF Sputtering", Fujitsu Sci. Tech. J. 8.1, pp. 161-81 (1972). 

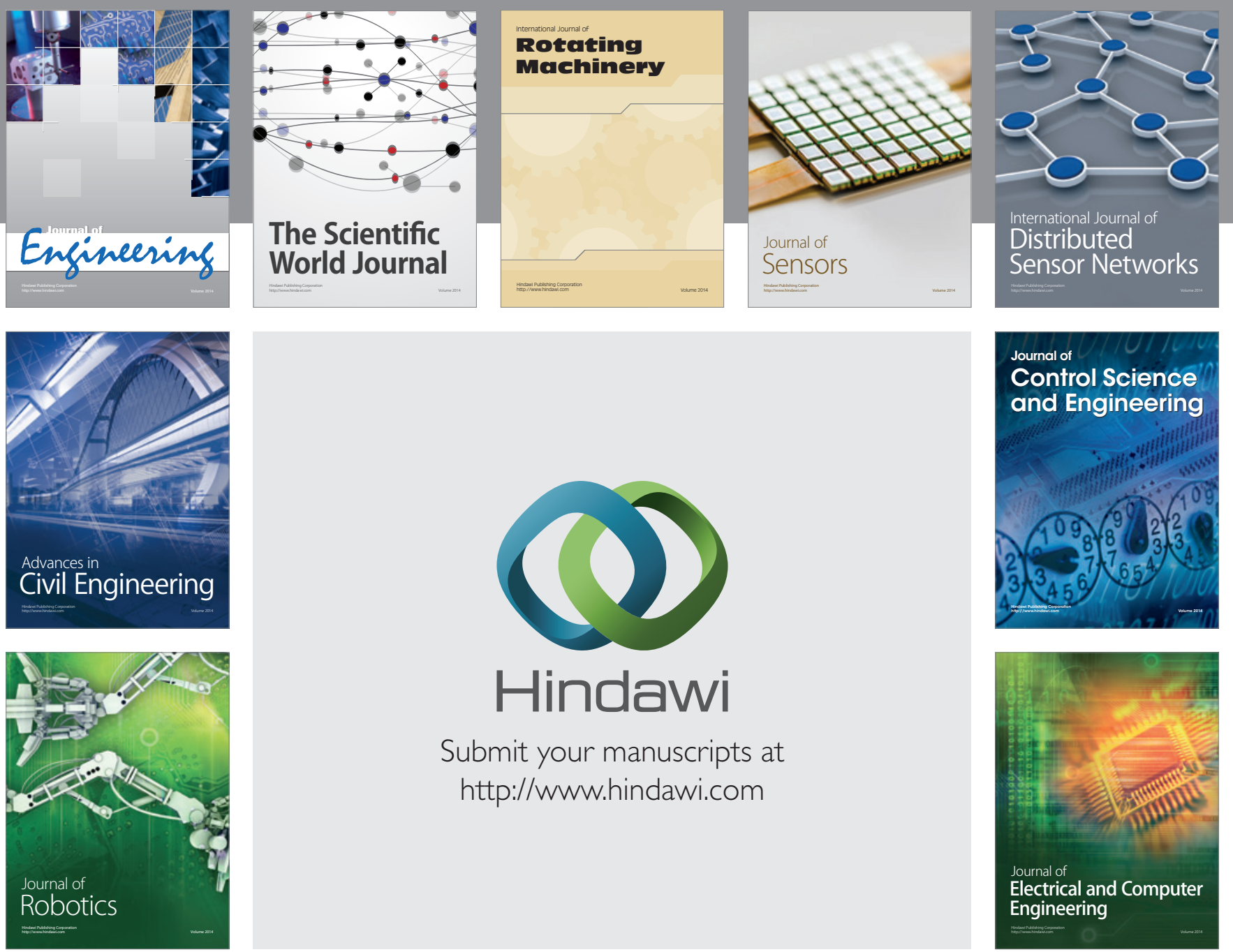

Submit your manuscripts at

http://www.hindawi.com
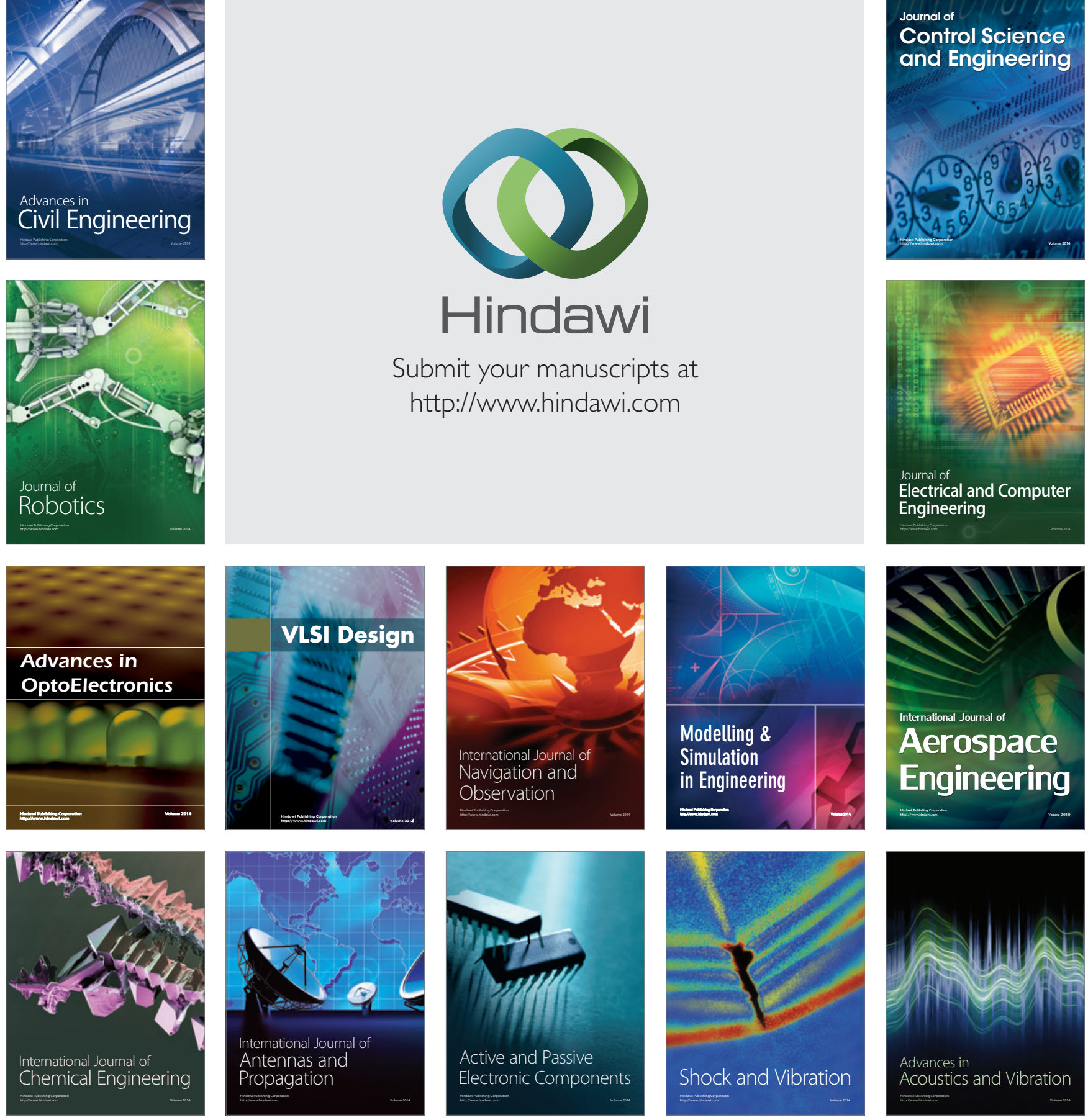